\title{
Paroxysmal autonomic instability with dystonia (PAID) syndrome following cardiac arrest
}

\author{
Dheeraj Kapoor ${ }^{1}$, MD, FCCP, Deepak Singla ${ }^{1}$, MD, Jasveer Singh ${ }^{1}$, MD, Rohit Jindal' ${ }^{2}$, MS
}

\begin{abstract}
Paroxysmal autonomic instability with dystonia (PAID) appears to be a unique syndrome following brain injury. It can echo many life-threatening conditions, making its early recognition and management a challenge for intensivists. A delay in early recognition and subsequent management may result in increased morbidity, which is preventable in affected patients. Herein, we report the case of a patient who was diagnosed with PAID syndrome following prolonged cardiac arrest, and discuss the pathophysiology, clinical presentation and management of this rare and under-recognised clinical entity.
\end{abstract}

Keywords: autonomic nervous system, brain injury, cardiac arrest, diffuse axonal injury, paroxysmal autonomic instability with dystonia

\section{INTRODUCTION}

Autonomic dysregulation (dysautonomia), which is a risk factor for increased morbidity in patients with brain injury, can challenge patients' acute management and their rehabilitation phase. ${ }^{(1)}$ Early recognition and treatment can reduce long-term disability and may result in better clinical outcome. Paroxysmal autonomic instability with dystonia (PAID) syndrome, a subset of dysautonomia, is a discrete clinical entity that can mimic certain other life-threatening conditions with similar presentations. Early recognition of this syndrome and its appropriate management may result in less expensive diagnostic tests, avoidance of pharmacological overdoses, prevention of secondary injury, enhanced nursing care and alleviation of anxiety among healthcare personnel observing periodic episodes in affected patients. ${ }^{(1,2)}$ The essential features of this characteristic syndrome include paroxysms of marked agitation, diaphoresis, hyperthermia, hypertension, tachycardia and tachypnoea accompanied by hypertonia and extensor posturing. ${ }^{(2,3)}$ PAID syndrome mainly manifests in the intensive care setting but may persist for months during the patient's rehabilitation phase. In this report, we present the case of a patient who was diagnosed with PAID syndrome following prolonged cardiac arrest, and discuss the pathophysiology, clinical presentations and management of this rare and under-recognised clinical entity.

\section{CASE REPORT}

A 26-year-old man presented to the trauma resuscitation room at the Government Medical College and Hospital, Chandigarh, India, after a fall from a height of $10 \mathrm{~m}$. During initial assessment and physical examination (Advanced Trauma Life Support [ATLS ${ }^{\circledR}$ ] protocol), there were no clinical signs of life-threatening injuries. Initial neurological examination revealed paraparesis, with loss of pain and temperature sensation below the level of T4 dermatome. Cranial nerve functions and higher mental functions were found to be normal. Plain radiography and computed tomography (CT) of the spine in the anteroposterior and lateral views revealed a compression fracture of the T4 and T5 vertebrae.
A spinal fusion surgery via the anterolateral approach under general anaesthesia was planned by the orthopaedic surgeons. In the operating room, the patient was premedicated with glycopyrrolate and fentanyl. Anaesthesia was induced using intravenous (IV) propofol and tracheal intubation facilitated by IV vecuronium bromide. Anaesthesia was maintained using a rate-controlled IV infusion of propofol, vecuronium and fentanyl with 50\% oxygen in the air. During the surgical intervention, a blood loss of $3.5 \mathrm{~L}$ occurred, which was managed well using fluids and blood products. The surgery lasted for approximately six hours and the patient was haemodynamically stable throughout the surgical procedure. Postoperatively, the patient was shifted to the intensive care unit (ICU) owing to hypothermia, and was extubated after six hours.

On Day 2 in the ICU, the patient developed difficulty in breathing and excessive cough with expectoration. On auscultation, there were coarse crepitations in the right middle zone of the chest. Chest radiography showed a heterogeneous opacity in the right middle zone. Suspecting pneumonia, tracheal aspirate was sent for culture and the patient was started on empirical broad-spectrum antibiotics (co-amoxiclav $1.25 \mathrm{~g} \mathrm{q} 8$ hours and amikacin $500 \mathrm{mg}$ q 12 hours IV). Noninvasive ventilation was initiated on the bilevel positive airway pressure mode.

On Day 3 of ICU stay, the patient's condition deteriorated further. He became dyspnoeic and drowsy, and arterial blood gas analysis showed severe respiratory acidosis. His trachea was immediately intubated and the ventilatory support adjusted to the synchronised intermittent mandatory ventilation (SIMV [VC]) mode. He had a sudden episode of pulseless ventricular tachycardia (VT). Cardiopulmonary resuscitation was initiated and the patient was successfully revived after 10 minutes. Severe hyperkalaemia was found to be the precipitating cause of VT and immediate corrections were made. After successful resuscitation following cardiac arrest, the patient was kept on full ventilatory support (SIMV [VC] mode).

On Day 4 of ICU stay, the patient's consciousness level improved to 9 on the Glasgow Coma Score. On Day 5, ventilatory 
support was reduced, as he was able to take a few respiratory efforts although his sensorium was still altered. No muscle rigidity or autonomic instability was observed during this period. Later, his chest condition and sensorium improved, and he was found to be comfortable on the ventilator.

On Day 6 in the ICU, the patient suddenly became restless and anxious. He had paroxysms of severe hypertension, tachycardia and diaphoresis during which he became tachypnoeic and developed high-grade fever. He also had generalised dystonic movements that subsided spontaneously after the paroxysms. On average, the patient had 2-3 paroxysms a day, each lasting approximately 30 minutes. A provisional diagnosis of PAID syndrome was made based on the typical clinical symptoms observed in the patient and investigated further.

$\mathrm{CT}$ and magnetic resonance (MR) imaging were done during the patient's ICU stay (on the tenth day after cardiac arrest) after his clinical condition was stable. CT showed slightly diffuse hypodensities in the periventricular white matter, with normal grey/white matter interface. MR images showed mild cerebral atrophy with ventricular dilatation that was consistent with CT findings. These changes were suggestive of hypoxic insult to the brain during the prolonged resuscitation efforts, ${ }^{(4)}$ and were probably the cause of the discrete symptoms and signs evident in the patient.

In the meantime, the patient was tracheostomised due to difficulty in weaning and kept on the SIMV (VC) mode of ventilation. Symptomatic management of the varied symptoms seen during paroxysms was started. Propranolol (10 mg q 8 hours via nasogastric tube) and clonidine infusion (20-50 $\mu \mathrm{g} /$ hour IV) was initiated to control the sympathetic symptoms of PAID syndrome, with dramatic positive response. It was, however, difficult to control hyperthermia, which responded poorly to paracetamol. Diclofenac sodium (75 mg q 8 hours IV) and hydrotherapy were started, and the patient responded well. Dystonic movements were controlled by intermittent boluses of midazolam (2 mg IV). Continuous rate-controlled infusion of morphine $(0.02 \mathrm{mg} / \mathrm{kg} / \mathrm{hour})$ and midazolam $(0.05 \mathrm{mg} / \mathrm{kg} / \mathrm{hour})$ was then started. Three days after the initiation of treatment, the patient responded well and was shifted to the high dependency unit (HDU) while using a T-piece with a tracheostomy tube in situ.

The patient was kept in the HDU for five days and in a rehabilitation ward for an additional two weeks. During his stay in the HDU, the clonidine infusion was gradually weaned off. Occasional surges of autonomic arousal, which were observed during the patient's stay even during the rehabilitation phase, were satisfactorily controlled by oral propranolol. Oral propranolol was continued after his discharge from the hospital. Dystonic movements and muscle rigidity, which were a matter of concern during the rehabilitation phase, responded initially to continuous infusion of morphine and midazolam only. Later, the infusions were switched to oral lorazepam ( 2 mg q 8 hours) with satisfying results. The medication was also prescribed following discharge.

\section{DISCUSSION}

PAID syndrome presents in patients with severe brain injury primarily due to trauma or hypoxia resulting in diffuse axonal or brainstem injury. ${ }^{(1,3)}$ It may also manifest in patients with intracranial haemorrhage, tumours or hydrocephalus. ${ }^{(3)}$

Dysregulation of the autonomic nervous system following brain injury may elucidate the genesis of PAID syndrome. ${ }^{(5,6)}$ Bullard suggested that it might be caused by damage to the cortical and subcortical areas, which control the vegetative functions (including regulation of blood pressure and temperature). ${ }^{(6)}$ Boeve et al, who elaborated on the concept, speculated that PAID syndrome might be the result of the activation or disinhibition of central sympathoexcitatory regions in the brain. ${ }^{(7)}$

The diagnosis and management of PAID syndrome is mainly based on the correct interpretation of a patient's clinical manifestations. The clinical signs invariably observed in PAID syndrome include elevated temperature $\left(\geq 38.5^{\circ} \mathrm{C}\right)$, tachycardia ( $\geq 130$ beats per minute), tachypnoea ( $\geq 140$ breaths per minute), agitation (Rancho Los Amigos Scale level $\leq \mathrm{IV}$ ), diaphoresis and dystonia (rigidity or decerebrate posturing).(2) The duration of an episode is cyclic, with at least one cycle per day for at least three days. ${ }^{(2)}$ The onset of these typical clinical signs generally occurs in the first week after brain injury and can persist for weeks or months, particularly in patients with hypoxic injuries. Due to the episodic nature of these manifestations, electroencephalography findings may be difficult to obtain and coordinate. Also, creatine phosphokinase level may or may not be elevated in PAID syndrome. ${ }^{(2)}$

The differential diagnosis of PAID syndrome includes other clinical entities such as sepsis, delirium tremens, dystonia, meningitis, neuroleptic malignant syndrome, thyroid storm and malignant hyperthermia, ${ }^{(2)}$ and clinicians should be mindful that the treatment approaches are altogether different for these various illnesses. While sepsis can mimic PAID syndrome, the paroxysmal nature of symptoms such as autonomic activation and generalised dystonia may not be present distinctively in patients with sepsis. In our patient, there were 2-3 paroxysms of symptoms that lasted for approximately 30 minutes and subsided spontaneously. The patient was free of any symptoms or signs in between the paroxysms. The paroxysmal nature of symptoms in our patient thus points toward PAID syndrome.

Similarly, malignant hyperthermia may also mimic PAID syndrome. The hypermetabolic state is usually triggered following exposure to certain drugs such as non-depolarising agents and inhalational volatile agents during the intraoperative period. (2) The symptoms may be present during induction or within 2-3 hours and rarely within 24 hours of exposure. ${ }^{(2)}$ The possibility of malignant hyperthermia in our patient was ruled out, as he was not exposed to any pharmacological triggering agents during the intraoperative period and had no symptoms postoperatively for nearly six days in the ICU. ${ }^{(2)}$

The management of patients with PAID syndrome is usually symptom-based and they often need supportive care. Profound hyperthermia and diaphoresis are commonly seen in patients with PAID syndrome, and if uncontrolled, can complicate their fluid management. Thermoregulatory dysfunction leading to hyperthermia in these patients may be the result of hypothalamic dysfunction or the hypermetabolic state being induced by sustained muscular contractions. ${ }^{(2)}$ Patients with PAID syndrome 
usually undergo extensive evaluations in search of a probable cause for the hyperthermia, including multiple cultures from various specimen types. A negative result from such cultures should be taken to indicate a probable diagnosis of PAID syndrome. Antipyretics and nonsteroidal anti-inflammatory drugs produce limited success in these patients, ${ }^{(8)}$ and passive therapy with cooling blankets and hydrotherapy might offer more effective strategies for controlling hyperthermia. While dantrolene is also usually used as an adjunct for the cessation of hyperthermia, its therapeutic action has been reported to be via a peripheral mechanism that has no effect on the central pathway, and hence it might be ineffective for patients with PAID syndrome. ${ }^{(8)}$ Our patient had high-grade fever for which symptomatic relief was sought. However, he did not respond to intravenous paracetamol, and cultures of the blood and tracheal aspirate were found to be sterile. IV diclofenac sodium along with aggressive hydrotherapy was thereafter prescribed, with desired results. Sympathetic nervous system excitation appears to be a major clinical feature of PAID syndrome. ${ }^{(2)}$ A combination of propranolol and clonidine was prescribed for our patient for control of the sympathetic symptoms, with successful outcome. Drugs causing $\beta$-adrenergic blockade such as propranolol (nonselective $\beta$-adrenergic blockade) or labetalol hydrochloride (nonselective $\beta$ - and selective $\alpha 1$-adrenergic blockade) are pertinent choices with proven clinical efficacy for the amelioration of sympathetic symptoms (such as hypertension and tachycardia). ${ }^{(9)}$ Selective $\beta 1$-adrenergic antagonists (such as metoprolol or atenolol) are not as effective in alleviating the autonomic response. ${ }^{(10)}$ Clonidine ( $\alpha 2$-adrenergic agonist), on the other hand, not only controls hypertension but also has a sedative and behaviour-stabilising effect by way of interrupting the feedback into the system. ${ }^{(2)}$ Anecdotal reports recommend the use of intrathecal baclofen ( $\gamma$-aminobutyric acid [GABA]-B receptor agonist) ${ }^{(11)}$ and oral gabapentin (GABA analogue $)^{(12)}$ for alleviating autonomic dysfunction following severe brain injury. These drugs may act by normalising the afferent stimuli in dysautonomic patients. However, the abovementioned drugs were not prescribed for our patient, as he responded well to our drug regimen (propranolol and clonidine), probably due to the lesser severity of brain insult. During the stay in the HDU and rehabilitation ward, clonidine infusion was successfully weaned off for our patient. However, propranolol was continued until discharge for adequate control of potential autonomic surges.

In our patient, episodic generalised dystonic movements that subsided on their own were observed only during the paroxysmal period. These dystonic movements, if not managed in a timely manner, can result in generalised motor rigidity and pose difficulties during supportive nursing care in the ICU. A search of the literature showed that various drugs such as morphine, ${ }^{(1,6)} \alpha$ or $\beta$ blockers, ${ }^{(3,9,10)}$ dopamine agonists ${ }^{(6,7,13)}$ and benzodiazepines ${ }^{(3,14)}$ could be used to alter central nervous system responsiveness by virtue of changes to $\alpha, \beta, \mathrm{GABA}$, dopamine or opiate receptors, respectively. As the dystonic movements subsided in our patient when intermittent boluses of IV midazolam were given, continuous IV infusion of midazolam along with morphine was administered to control the abnormal movements, with good results. Morphine was added to the midazolam infusion to make use of their synergistic effects, as studies have suggested that, anatomically, there is extensive superimposition of opiate receptor areas with the dopaminergic systems, and therefore that a structural lesion in these anatomical areas would conceivably affect both systems. ${ }^{(15)}$ During the rehabilitation phase and upon discharge, the infusions were replaced by oral lorazepam for our patient. Lorazepam, in addition to its anxiolytic and sedative effects, also has a muscle relaxant property and may be beneficial in patients with PAID syndrome. ${ }^{(2)}$ Drugs such as chlorpromazine or phenobarbital should be avoided, as their usefulness in these patients is not yet proven. ${ }^{(7)}$

At present, although the assorted symptoms of PAID syndrome do form a discernable pattern, clinicians do not completely understand the basic mechanisms underlying hyperthermia, hypertension and muscular rigidity in affected patients. Future research into the pathophysiology of the variable symptoms and signs seen in patients with PAID syndrome may facilitate earlier recognition and more logical and cogent management, thereby helping to reduce associated morbidity and mortality.

\section{REFERENCES}

1. Baguley IJ, Nicholls JL, Felmingham KL, et al. Dysautonomia after traumatic brain injury: a forgotten syndrome? J Neurol Neurosurg Psychiatry 1999; 67:39-43.

2. Blackman JA, Patrick PD, Buck ML, Rust RS Jr. Paroxysmal autonomic instability with dystonia after brain injury. Arch Neurol 2004; 61:321-8.

3. Pranzatelli MR, Pavlakis SG, Gould RJ, De Vivo DC. Hypothalamicmidbrain dysregulation syndrome: hypertension, hyperthermia, hyperventilation, and decoration. J Child Neurol 1991; 6:115-22.

4. Bulakbasi N, Pabuscu Y, Kurtaran HK, et al. "Results of Acute Cerebral Anoxia in Adults: is it a Reversal Sign?" Turk J Med Sci 2000; 30:571-77.

5. Baguley IJ, Heriseanu RE, Cameron ID, Nott MT, Slewa-Younan S. A critical review of the pathophysiology of dysautonomia following traumatic brain injury. Neurocrit Care 2008; 8:293-300.

6. Bullard DE. Diencephalic seizures: responsiveness to bromocriptine and morphine. Ann Neurol 1987; 21:609-11.

7. Boeve BF, Wijdicks EF, Benarroch EE, Schmidt KD. Paroxysmal sympathetic storms ("diencephalic seizures") after severe diffuse axonal head injury. Mayo Clin Proc 1998; 73:148-52.

8. McEvoy GK (Ed). American Hospital Formulary Service Drug Information, American Society of Hospital Pharmacists. Bethesda, 1994:1195-286

9. Chiolero RL, Breitenstein E, Thorin D. Effects of propranolol on resting metabolic rate after severe head injury. Crit Care Med 1989; 17:328-34.

10. Do D, Sheen VL, Bromfield E. Treatment of paroxysmal sympathetic storm with labetalol. J Neurol Neurosurg Psychiatry 2000; 69:832-3.

11. Becker R, Benes L, Sure U, Hellwig D, Bertalanffy H. Intrathecal baclofen alleviates autonomic dysfunction in severe brain injury. J Clin Neurosci 2000; 7:316-9.

12. Baguley IJ, Heriseanu RE, Gurka JA, Nordenbo A, Cameron ID. Gabapentin in the management of dysautonomia following severe traumatic brain injury: a case series. J Neurol Neurosurg Psychiatry 2007; 78:539-41.

13. Russo RN, O'Flaherty S. Bromocriptine for the management of autonomic dysfunction after severe traumatic brain injury. J Paediatr Child Health 2000; 36:283-5.

14. Silver JK, Lux WE. Early onset dystonia following traumatic brain injury. Arch Phys Med Rehabil 1994; 75:885-8.

15. Granato JE, Stern BJ, Ringel A, Karim AH, et al. Neuroleptic malignant syndrome: successful treatment with dantrolene and bromocriptine. Ann Neurol 1983; 14:89-90. 